\title{
In Vitro Antimicrobial and Antioxidant Activity of Some Berry Species
}

Tuğba İduğ ${ }^{1 *}$, Hilal Hızlı², Ali Şen ${ }^{3}$, Fatma Koç

1 Istanbul Medipol University, School of Pharmacy, Department of Pharmacognosy, Istanbul, Turkey.

2 Istanbul Medipol University, Faculty of Health Sciences, Department of Nutrition and Dietetics, Istanbul, Turkey. 3 Marmara University, Faculty of Pharmacy, Department of Pharmacognosy, Istanbul, Turkey.

4 Istanbul Medipol University, International School of Medicine, Department of Clinical Microbiology, Istanbul, Turkey.

\section{ABSTRACT}

The aim of this work was to determine antioxidant and antimicrobial activities of extracts obtained from fresh and dried fruits of Vaccinium macrocarpon, Morus nigra, Fragaria $X$ ananassa. Antioxidant and antimicrobial activity of extracts were assayed by DPPH and disc diffussion methods. Antioxidant activity of extracts decreased in the following order: Fragaria X ananassa fresh fruit (FAF) $>$ Vaccinium macrocarpon fresh fruit (VMF) $>$ Morus nigra fresh fruit (MNF) $>$ Morus nigra (MND2) > Morus nigra $($ MND1) $>$ Vaccinium macrocarpon $($ VMD1) $>$ Fragaria $X$ ananassa $($ FAD1) $>$ Vaccinium macrocarpon $(V M D 2)>$ Fragaria $X$ ananassa (FAD2). Fresh fruits showed higher antioxidant activity than dried fruits. FAF showed highest antimicrobial activity aganist $E$. coli and MND2 showed higher antimicrobial activity aganist $E$. coli and $S$. aureus in comparison to other extracts. Other extracts showed nearly same antimicrobial activity. All extracts showed no antimicrobial activity aganist $C$. albicans.

Keywords: Mulberry, Strawberry, Cranberry, Antioxidant activity, Antimicrobial activity

\section{INTRODUCTION}

Dietary patterns characterized by relatively high intakes of fruits and vegetables are consistently associated with reductions in the incidence of noncommunicable diseases such as coronary heart disease, stroke, cancer, and various chronic disease $^{1}$. Berries provide significant health benefits because of their high levels of polyphenols, antioxidants, vitamins, minerals, and fibers ${ }^{2}$. Most berries are 
delicious and powerful disease-fighting foods and make up the largest proportion of fruit that is consumed in the human diet. Berry fruits are popularly consumed not only in fresh and frozen forms but also as processed and derived products, including dried ${ }^{3}$.

Oxidative stress plays an important role in the pathogenesis of most chronic diseases ${ }^{4}$. ROS is said to play an important role in many chronic diseases such cardiovascular diseases, diabetes, inflammation, anaemia, degenerative diseases, cancer ${ }^{5}$. Antioxidants molecules had defensive effects against reactive oxygen species (ROS) in the body. The plants have been known since ancient times as a good antioxidant ${ }^{4}$. Fruits rich in antioxidants can prevent or delay oxidative damage ${ }^{6}$. Fresh fruits are rich in acids, also contain anthocyanins and flavonoids 7 . Anthocyanins and flavonoids have been identified as strong antioxidant ${ }^{8}$. Especially, berry fruits worldwide known and consumed have been well studied Berries, including raspberries, blueberries, black currants, red currants, and cranberries are a rich source of dietary antioxidant ${ }^{1}$.

Vaccinium macrocarpon known as cranberry naturally grows North America. Fresh fruits are rich in acids, also contain anthocyanins and flavonoids ${ }^{7}$. Anthocyanins and flavonoids have been identified as strong antioxidant ${ }^{8}$. Morus nigra known as mulberry is native to southwestern Asia also cultivated so long time and natural origin is unknown. Mulberries contain vitamins, minerals and anthocyanins 9 . Fragaria $X$ ananassa is cultivated variety of strawberries $^{10}$. Strawberries contain various phenolic compounds such as hydroxycinnamic acids, ellagic acid, ellagitannins, flavan-3-ols, flavonols, and anthocya$\operatorname{nin} s^{11}$.

Certain berries rich in tannins have been found to increase bacterial infections. Among the berries, cranberries, cloudberries, red raspberries, strawberries, and bilberries possess clear antimicrobial effects against human pathogens. Berry ellagitannins are strong antimicrobial agents acting as possible antiadherence compounds in preventing the colonization and infection of many pathogens. Several mechanisms of action in the inhibition of bacteria are involved, such as destabilization of cytoplasmic membrane, permeabilization of plasma membrane, inhibition of extracellular microbial enzymes, direct actions on microbial metabolism, and deprivation of the substrates required for microbial growth ${ }^{12,13}$. However, there is very little information about the antimicrobial capacity of phenolics present in berries, except in cranberry ${ }^{3}$.

The aim of this study was to determine antioxidant and antimicrobial activities of extracts obtained from fresh and dried fruits of Vaccinium macrocarpon, Morus nigra, Fragaria X ananassa.

\section{METHODOLOGY}

\section{Plant Material}

Fresh fruits and two different dried fruits of Vaccinium macrocarpon, Morus nigra and Fragaria X ananassa were purchased from different local markets (Table 1).

Table 1. List of sample and abbreviations used in this study

\begin{tabular}{|c|c|c|c|}
\hline Botanical name & Fragaria X ananassa & $\begin{array}{c}\text { Vaccinium } \\
\text { macrocarpon }\end{array}$ & Morus nigra \\
\hline Fresh fruits & FAF & VMF & MNF \\
\hline Dried fruits 1 & FAD1 & VMD1 & MND1 \\
\hline Dried fruits 2 & FAD2 & VMD2 & MND2 \\
\hline
\end{tabular}

\section{Chemicals}

Methanol, ethanol, 2,2-diphenyl-1-picrylhydrazyl (DPPH) were provided from Sigma (Steinheim, Germany). Müeller Hinton agar, Saubaroud Dextrose broth were provided from Merck (Darmstadt, Germany).

\section{Extraction of Plant Material}

All fruit samples (100 g) were ground in a grinder and macerated with methanol (200 $\mathrm{ml}$ ) for four days at room temperature and the extracts were filtered. Then methanol was evaporated with rotary evaporator. All extracts were stored in refrigerator at $4 \mathrm{C}^{\mathrm{o}}$ until use.

\section{DPPH Radical Scavenging Activity}

The ability to scavenge DPPH radical of extracts was determined according to the method of Yanping Zou ${ }^{14}$. Briefly stock extracts were prepared at $10 \mathrm{mg} /$ $\mathrm{mL}$ concentration and diluted to $2,5 \mathrm{mg} / \mathrm{mL}, 0,625 \mathrm{mg} / \mathrm{mL}, 0,156 \mathrm{mg} / \mathrm{mL}$ with methanol. $10 \mu \mathrm{L}$ of all dilutions were added $190 \mu \mathrm{L}$ DPPH solution in a well of 96 well-plate. The mixture was shaken quietly and left in room temperature and dark for 30 minutes. After then the absorbance was measured aganist methanol using a microplate reader at $517 \mathrm{~nm}$.

DPPH radical scavenging activity were calculated according to following:

Antioxidant activity $(\%)=\left[\left(\mathrm{A}_{0}-\mathrm{A}_{1}\right) / \mathrm{A}_{0}\right] \mathrm{X} 100$

Where $\mathrm{A}_{\mathrm{o}}$ is the absorbance of control, $\mathrm{A} 1$ is the absorbance of extracts/standard. Extract concentration providing $50 \%$ inhibition $\left(\mathrm{IC}_{50}\right.$ ) was calculated from the graph plotting inhibition percentage aganist extract concentration. Test were carried out in dublicated. Ascorbic acid was used as positive control. 


\section{In vitro Antimicrobial Activity Assay}

In this study, disk diffusion method was used to determine of antimicrobial activity of the berries. This method is used for detection whether the samples have inhibition effect on microorganisms ${ }^{15}$. Also used for determination effects of drug and comparison of standards ${ }^{16}$.

\section{Microbial Strains And Growth Conditions}

The assessment of antimicrobial activity was performed on gram positive bacteria Staphylococcus aureus ATCC 25923, gram negative bacteria Echerichia coli ATCC 25922 and yeast Candida albicans ATCC 10231 was determined by the disc diffusion method. Bacterial cultures were grown at $370 \mathrm{C}$ for 24 hours in Brain Heart Inhibition broth or agar (BHB, BHA, Merck, Darmstadt, Germany), yeast strain was grown at $30 \mathrm{oC}$ for 48 hours in Saubaroud Dextrose broth or agar (SDB, SDA, Merck, Darmstadt, Germany). Microbial cultures for antimicrobial testing were prepared by picking colony from 24 or 48-h-old BHA/SDA

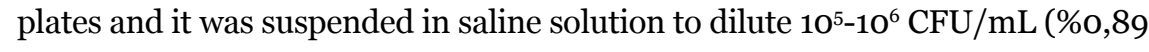
$\mathrm{NaCl}$ ). The disk diffusion method was performed on Müeller Hinton agar (MHA, Merck, Darmstadt, Germany) for bacterial strains and SDA for yeast strain.

\section{Disk Diffusion Method}

Each of extracts were diluted in sterile distilled water (o,1 w/v). For the disk diffussion assay $0,1 \mathrm{~mL}$ of each microbial suspension was spread on a solid growth medium in a Petri dish. Three sterile paper disk (6 $\mathrm{mm}$ diameter) were impregnated with $15 \mathrm{uL}$ each plant extract solution and were placed on the surface of agar plate. Plates were incubated for appropriate conditions for microbial strains. Antimicrobial activity was determined with inhibition zone around the disk following incubation. Impregnated discs with ethanol used as positive control ${ }^{17}$.

\section{Minimum Inhibitory Concentration (MIC) Assay}

Minimum inhibitory concentration (MIC) is described as the lowest concentration of antimicrobial agent is needed to kill the bacteria ${ }^{18}$. MIC of all extracts were determined by microdilution techniques in Mueller-Hinton broth (MHB) for bacteria. Inoculates prepared in the MHB at a density adjusted to 0,5 McFarland turbidity standard and diluted $1 / 10$ for the broth microdilution procedure ${ }^{15}$. The data were given as means \pm standard deviations and analysed by one-way analysis of variance (ANOVA) followed by the Tukey's multiple comparison tests using GraphPad Prism.

\section{RESULTS AND DISCUSSION}

Our focus in this study was to complement the previous knowledge of antioxi- dant and antimicrobial activities of fresh and dry samples of berries.

The antioxidant power of fruit is closely correlated to the presence of efficient oxygen radical scavengers, such as vitamin $\mathrm{C}$ and phenolic compounds ${ }^{19}$. Berries are consistently ranked among the top sources of total phenolics and TAC, with levels up to 4 times greater than other fruits, 10 times greater than vegetables ${ }^{20}$. In a study by Tulipani et al. individual contribution was investigated in different strawberry cultivars, where vitamin $\mathrm{C}$ was found to be one of the most important components responsible for more than $30 \%$ of the TAC of strawberry extracts, followed by anthocyanins contributing $25 \%$ to $40 \%^{21}$. Viskelis and others reported that significantly larger amounts of anthocyanins were determined in the overripe cranberries of the cultivars ${ }^{22}$. Similarly, antioxidant activity in fresh strawberry was found to be highest in our study, followed by fresh cranberry and mulberry extracts.

A low $\mathrm{IC}_{50}$ value (the concentration of extract, which is required to scavenge $50 \%$ of DPPH free radical) means strong antioxidant activity. FAF showed the highest antioxidant activity with $\mathrm{IC}_{50}$ value of $0,327 \mathrm{mg} / \mathrm{mL}$, while FAD2 showed the lowest antioxidant activity with $\mathrm{IC}_{50}$ value of $3,331 \mathrm{mg} / \mathrm{mL}$ in DPPH assay. All extracts showed low antioxidant activity compared to standard. It was determined that fresh extracts showed higher antioxidant activity. Antioxidant activities of extracts decreased in the following order: $\mathrm{FAF}>\mathrm{VMF}>\mathrm{MNF}>\mathrm{MND} 2>\mathrm{MN}$ D1 $>$ VMD1 $>$ FAD1 $>$ VMD2 $>$ FAD2 (Table 2).

It was thought that, drying and storage conditions may have affected the antioxidant capacity of dry extracts. In the phytochemical studies on these plants, it have been reported that this species contained phenolic compounds such as flavonoids and anthocyanins intensively. Therefore, the antioxidant activity of these fruits might be resulting from the phenolic contents of them. 
Table 2. Antioxidant activities of extracts

\begin{tabular}{|c|c|}
\hline Extracts / Standards & $\begin{array}{c}\text { DPPH activity } \\
\mathbf{I C}_{\mathbf{5 0}} \text { ( } \mathbf{m g m L}^{-1} \mathbf{)}\end{array}$ \\
\hline FAF & $0,327 \pm 0,002 \mathrm{c}$ \\
\hline FAD2 & $3,331 \pm 0,024 \mathrm{j}$ \\
\hline FAD1 & $2,189 \pm 0,005 \mathrm{~h}$ \\
\hline VMF & $0,408 \pm 0,007 \mathrm{~cd}$ \\
\hline VMD2 & $2,332 \pm 0,054 \mathrm{i}$ \\
\hline VMD1 & $1,640 \pm 0,021 \mathrm{~g}$ \\
\hline MNF & $0,454 \pm 0,005 \mathrm{~d}$ \\
\hline MND2 & $1,145 \pm 0,045 \mathrm{e}$ \\
\hline MND1 & $1,318 \pm 0,004 \mathrm{f}$ \\
\hline Ascorbic acid & $0,002 \pm 0,000 \mathrm{a}$ \\
\hline Butylated hydroxyanisole & $0,057 \pm 0,000 \mathrm{~b}$ \\
\hline
\end{tabular}

Low $\mathrm{IC}_{50}$ value indicates high antioxidant activity.

Each value in the table is represented as mean $\pm \operatorname{SD}(n=3)$

Different letter superscripts in the same column indicate significant differences $(\mathrm{P}<0.05)$

In this study, disc diffusion method was used to determination of antimicrobial activity of strawberries, blueberries and black mulberies on E. coli ATCC 25922, S. aureus ATCC 25923 and C. albicans ATCC 10231. Inhibition zones were measured and shown in Table 3.

This study showed that, dry strawberry (FAD2) has the highest level of antimicrobial activity was observed against $E$. coli. There was no inhibition zone against $C$. albicans. The dry blueberry (VMD2) has inhibition zone of $7 \mathrm{~mm}$ diameter against S. aureus.

In addition to disk diffusion method, broth dilution method was also performed to observe effect against on E. coli and S. aureus. Fresh strawberry (FAF), fresh blueberry (VMF) and dry black mulberry (MND1) were found that most effective on $S$. aureus. Dry black mulberry (MND1) was also found that most effective extract against to E. coli (Table 4).

One of the study showed that the highest antimicrobial activity of blueberry against E. coli and it has $18,67 \pm 1,15 \mathrm{~mm}$ inhibition zone also the lowest antimicrobial activity was found that against $S$. aureus and has $11,00 \pm 2,00 \mathrm{~mm}$ diameter ${ }^{23}$. Howell reported that high-molecular weight proanthocyanidins (condensed tannins) from cranberry juice inhibit the adherence of uro-pathogenic fimbriated $E$. coli and thus offer protection against urinary tract infections ${ }^{24}$. Compared with our study, it was observed that blueberries did not produce any antimicrobial product against $S$. aureus and C. albicans, which showed that higher antimicrobial activity against $E$. coli. Another study showed that blueberry inhibited the growth of $E$. coli and $S$. aureus, but did not inhibit the $C$. albicans ${ }^{25}$. It was shown that, Black mulberry has more effectively inhibition against to Gram positive bacteria than Gram negative bacteria ${ }^{26}$. All of the extracts did not have any effect on the growth of the yeast species (C. albicans) studied.

Table 3. Inhibition zone around disks

\begin{tabular}{|c|c|c|c|}
\hline Extracts / Control & E. coli & S. aureus & C. albicans \\
\hline Ethanol & $9 \mathrm{~mm}$ & $9 \mathrm{~mm}$ & $10 \mathrm{~mm}$ \\
\hline FAF & $6 \mathrm{~mm}$ & - & - \\
\hline FAD2 & $10 \mathrm{~mm}$ & - & - \\
\hline FAD1 & $11 \mathrm{~mm}$ & - & - \\
\hline VMF & $10 \mathrm{~mm}$ & - & - \\
\hline VMD2 & $9 \mathrm{~mm}$ & - & - \\
\hline VMD1 & $9 \mathrm{~mm}$ & - & - \\
\hline MNF & - & - & - \\
\hline MND2 & $8 \mathrm{~mm}$ & $7 \mathrm{~mm}$ & - \\
\hline MND1 & $9 \mathrm{~mm}$ & - & - \\
\hline
\end{tabular}

Table 4. MIC results

\begin{tabular}{|c|c|c|}
\hline \multirow{2}{*}{ Extracts/Control } & \multicolumn{2}{|c|}{ MIC $(\mathbf{m g} / \mathbf{m L})$} \\
\cline { 2 - 3 } & S. aureus & E. coli \\
\hline Ethanol & 0,1 & 0,1 \\
\hline MNF & 0,003125 & 0,05 \\
\hline FAF & 0,0015625 & 0,05 \\
\hline VMF & 0,0015625 & - \\
\hline MND2 & 0,00625 & 0,0125 \\
\hline FAD2 & 0,0125 & 0,1 \\
\hline VMD2 & 0,0125 & 0,05 \\
\hline MND1 & 0,0015625 & 0,00625 \\
\hline FAD1 & 0,003125 & 0,1 \\
\hline VMD1 & 0,05 & - \\
\hline
\end{tabular}


In conclusion, fresh samples of fruits showed higher antioxidant and antimicrobial capacity than dried samples. Strawberries also showed higher effects than other berry samples. The antioxidative and antimicrobial activity depends on the cultivar, growth conditions, storage of raw material, and the method of isolation of active substances. Drying can affect the amount and activity of antioxidant ingredients. Further studies are needed to verify the antioxidant and antimicrobial activity of the compounds of berries.

\section{ACKNOWLEDGMENTS}

We wish to acknowledge the excellent assistance of Ayşenur Çabuk, Beyza Geçer, Elif Zerek, Merve Sayın, Merve Aydoğdu who were students in Department of Nutrition and Dietetics, Istanbul Medipol University.

\section{REFERENCES}

1. Borges, G.; Degeneve, A.; Mullen, W.; Crozier, A. Identification of flavonoid and phenolic antioxidants in black currants, blueberries, raspberries, red currants, and cranberries. J. Agric. Food. Chem. 2010, 58, 3901-3909.

2. Zhao, Y.; Berry fruit value-added products for health promotion. CRC press, New York, 2007 , pp: 154-166.

3. Nile, S. H.; Park, S. W. Edible berries: Review on bioactive components and their effect on human health. Nutrition. 2013, 1-11.

4. Mahdi-Pour, B.; Jothy, S. L; Latha L.Y; Chen, Y; and Sasidharan S. Antioxidant activity of methanol extracts of different parts of Lantana camara, Asian. Pac. J. Trop. Biomed, 2012, 2(12), 960-965.

5. Kalı, P.; Gülçin, İ.; Gören, A. C. Antioxidant activity and polyphenol content of cranberries (Vaccinium macrocarpon) Rec. Nat. Prod. 2015, 9(4), 496-502.

6. Silva, K. D. R. R.; Sirasa M. S. F. Antioxidant properties of selected fruit cultivar grown in Sri Lanka. Food Chem. 2018, 238, 203-208.

7. Bruneton, J. Pharmacognosy Phytochemistry Medicinal Plants, 2nd ed. Lavoisier Publishing: Paris, 1999, pp: 363-364.

8. Yan, X.; Murphy, B. T.; Hammond, G. B.; Vinson, J. A.; Neto, C. C. Antioxidant activities and antitumor screening of extracts from cranberry fruit (Vaccinium macrocarpon). J. Agric. Food Chem. 2002, 50, 5844-5849.

9. Yiğit, D.; Mavi, A.; Aktaş, M. Antioxidant activities of black mulberry (Morus nigra). EUFBED. 2008, 1-2, 223-232

10. Aharoni, A.; Giri, A. P.; Verstappen, F. W. A.; Bertea, C. M.; Sevenier, R.; Sun, Z.; Jongsma, M. A.; Schwaab, W.; Bouwmeester, H. J. Gain and Loss of fruit flavor compounds prodused by wild and cultivated strawberry species. Plant Cell. 2oo4, 16, 3110-3131.

11. Oszmianski, J.; Wojdyto, A. Comparative study of phenolic content and antioxidant activity of strawberry puree, clear, and cloudy juices. Eur. Food. Res. Technol. 2009, 228, 623-631

12. Puupponen, P. R.; Nohynek, L.; Alakomi, H. L.; Aksman-Caldentey, K. M. Bioactive berry compounds novel tools aganist human pathogens. Appl. Microbiol. Biotechnol. 2005, 67, 8-18. 13. Puupponen, P. R.; Nohynek, L.; Hartmann-Schmidlin, S.; Kahkonen, M.; Heinonen, M.;
Maastta-Riihinen, K. et all. Berry phenolics selectively inhibit the growth of intestinal pathogens. J. Appl. Microbiol. 2005, 98, 991-1000.

14. Zou, Y.; Chang, S. K. C.; Gu, Y.; Qian, S. Y. Antioxidant activity and phenolic compositions of lentil (Lens culinaris var. Morton) extract and its fractions. J. Agric. Food Chem, 2011, 23 59(6), 2268-2276.

15. Pessini, G. L.; Filho, B. P. D.; Nakamura, C. S.; Cortez, D. A. G. Antibacterial activity of extracts and neolignans from Piper regnellii (Miq.) C. DC. var. pallescens (C. DC.) Yunck. Mems Inst. Oswaldo Cruz. 2003, 98(8), 1115-1120.

16. Alzoreky, N. S.; Nakahara, K. Antibacterial activity of extracts from some edible plants commonly consumed in Asia. Int. J. Food Microbiol. 2oo3, 80(3), 223-230.

17. Klancnik, A.; Piskernik, S.; Jersek, B.; Mozina, S. S. Evaluation of diffusion and dilution methods to determine the antibacterial activity of plant extracts. J. Microbiol. Methods. $\mathbf{2 0 1 0}$ 81(2), 121-126.

18. Balouiri, M.; Sadiki, M.; Ibnsouda, S. K. Methods for in vitro evaluating antimicrobial activity: A review, J. Pharm. Anal. 2016, 6, 71-79.

19. Giamperi, F.; Tulipani, S.; Alvares-Suarez, J. M.; Quiles, J.L.; Mezzetti, B.; Battino, M. The strawberry: Composition, nutritional quality, and impact on human health. Nutrition. 2012, $28,9-19$

20. Halvorsen, B. L.; Holte, K.; Myhrstad, M. C. W.; Barikmo, I, Hvattum, E.; Remberg, S. F; et al. A systematic screening of total antioxidants in dietary plants. J. Nutr. 2002, 132, 461-471.

21. Tulipani, S.; Mezzetti, B.; Capocasa, F.; Bompadre, S.; Beekwilder, J.; Ric de Vos C. H.; et al. Antioxidants, phenolic compounds, and nutritional quality of different strawberry genotypes. $J$ Agric. Food Chem. 2008, 56, 696-704.

22. Viskelis, P.; Rubinskiene, M.; Jasutiene, I.; Sarkinas, A.; Daubaras, R.; Cesoniene, L. Anthocyanins, antioxidative, and antimicrobial properties of Amerikan cranberry (Vaccinium macrocarpon Ait.) and their press cakes. J. Food Sci. 2009, 74(2), 157-161.

23. Bekki, S. Investigation of the antibacterial and cytotoxic effects of thyme oil, bilberry juice, cabbage juice and broccoli juice an in vitro conditions. 2010, Cumhuriyet University, Master of sicience thesis. Department of Microbiology, Sivas, Advisor: Zeynep Sümer (In Turkish).

24. Howell, A. B. Cranberry proanthocyanidins and maintenance of urinary tract health. Crit. Rev. Food Sci. Nutr. 2002, 42, 273-278.

25. Nohynek, L. J.; Alakomi, H.; Kähkönen, M. P.; Heinonen, M.; Ilkka, M.; R. H. Puupponenpimiä, R. H.; Helander, I. M. Berry Phenolics : Antimicrobial Properties and Mechanisms of Action Against Severe Human Pathogens. Nutr Cancer. 2006, 54(1), 18-32.

26. Khalid, N.; Fawad, S. A.; Ahmed, I. Antimicrobial activity, phytochemical profile and trace minerals of black mulberry (Morus Nigra L.) fresh juice. Pak. J. Bot. 2011, 43, 91-96. 